\title{
ECOLOGICAL
} MODELLING

\section{Dynamics of a fishery on two fishing zones with fish stock dependent migrations: aggregation and control}

\author{
R. Mchich ${ }^{\mathrm{a}, *}$, P.M. Auger ${ }^{\mathrm{b}}$, R. Bravo de la Parra ${ }^{\mathrm{c}}$, N. Raissi ${ }^{\mathrm{a}}$ \\ ${ }^{a}$ Université Ibn Tofail, Faculté des sciences, Laboratoire S.I.A.N.O., Dept. maths et info., B.P. 133, Kenitra, Morocco \\ ${ }^{\mathrm{b}}$ Laboratoire de Biometrie, Université C. Bernard de Lyon1, 43 Bd du 11 Novembre 1918, 69622 Villeurbanne Cedex, France \\ ${ }^{\mathrm{c}}$ Departamento de Matematicas, Universidad de Alcala, 28871 Alcala de Henares, Spain
}

Received 2 February 2001; received in revised form 23 October 2001; accepted 20 June 2002

\begin{abstract}
This work presents a specific stock-effort dynamic model. The stock corresponds to two fish populations growing and moving between two fishing zones, on which they are harvested by two different fleets. The effort represents the number of fishing vessels of the two fleets which operate on the two fishing zones. The bioeconomical model is a set of four ordinary differential equations governing the stocks and the fishing efforts in the two fishing areas. Fish migration, as well as vessels displacements, between the two zones are assumed to take place at a faster time scale than the variation of the stocks and the changes of fleets sizes, respectively. The vessels movements between the two fishing areas are assumed to be stock dependent, i.e. the larger the stock density is in a zone the more vessels tends to remain in it. The global fish stock and the total number of vessels keep constant at the fast time scale. This property enables, via aggregation methods, the reduction of the system dimension in order to proceed to its qualitative analysis. Under some assumptions, we obtain either a stable equilibrium or a stable limit cycle which involves large cyclic variations of the total fish stock and fishing effort. Finally, we introduce a control parameter to maintain the system at a sustainable equilibrium and thus avoiding the important fluctuations founded otherwise.
\end{abstract}

(C) 2002 Elsevier Science B.V. All rights reserved.

Keywords: Population dynamics; Stock-effort model; Time scales; Aggregation methods; Control

\section{Introduction}

The overfishing of marine resources leads inexorably to the extinction of fish stocks and indir-

\footnotetext{
* Corresponding author. Tel.: +212-37-377559; fax: +21237-372770

E-mail address: racmchich@yahoo.com (R. Mchich).
}

ectly to harmful consequences for the coastal states economies. See, for example, Millischer et al. (1999) where the study of the evolution of Brittany's industrial fleets shows an important decrease of their overall fishing power in the eighties for saithe, cod, haddock and whiting in the West of Scotland area. Regarding the sardine stock of the Atlantic southern coast of Morocco, 
Belvéze (1984) reviews important variations of the overall fish catch from 1940 to 1983; data exhibit, for the different fisheries, almost cyclic fluctuations with large and increasing amplitudes from 90 to $310 \mathrm{kt}$. An important point related to overfishing is the evolution of the fishing efficiency of fleets to find the fish stocks and to reach rapidly the abundant fishing areas (Gascuel et al., 1993). Another important issue involved in fisheries management is the differentiation of fishing zones of a coastal state. There is the artisan fishery which operates on 3 miles from the coast, the coastal fishery between 3 and 12 miles and the high sea fishery beyond 12 miles. The adjacent coastal state, who is the owner of the resource evolving in his Exclusive Economical Zone, is responsible for the management of the global fishery which is shared by the above mentioned different types fisheries. So, in order to control the situation, it is important to have a good knowledge of the global evolution of the resource and of the activity related to its exploitation. The fishery management authorities must deal with the possible fishery conflicts resulting for instance from the simultaneous exploitation of two fishing zones. Theoretically, each kind of fleet operates in its zone according to its own fishery characteristics. In practice, the fish stock does not remain in a given area and frequently moves between two adjacent zones. Consequently, the fishing vessels do not hesitate to cross the fuzzy boundary between two adjacent zones in order to increase their catch.

Many mathematical models have been developed to describe the dynamics of fisheries (Allen and Mc Glade, 1986; Quinn and Deriso, 1999; Haddon, 2001). The simplest models assume a logistic equation for the stock with a term of catch which is proportional to the actual stock and to a constant fishing effort, which corresponds for example to the number of vessels involved in the fishery (Schaefer, 1954; Beddington and May, 1977; Rotenberg, 1987). The next step is to add an equation for the fishing effort leading to LotkaVolterra types of fishery models (Schaefer, 1954; Allen and Mc Glade, 1986). In these preypredator models, the number of vessels decreases at a constant rate while increases due to increasing fish catch. Such simple models predict persistence of the fishery, corresponding to stabilization at a constant level of fish stock and number of vessels involved in the fishery. Many other aspects have been added in fishery models such as the age structure and the growth of the fish populations and effects of fluctuations (Beddington and May, 1977). External factors such as food, predators, light and habitat can also be considered in more complete models, (Mackinson, 2000) for herring shoals using a fuzzy expert system. More complex models explore the stability of marine ecosystems impacted by a fishery (Vasconcellos et al., 1997).

The inclusion of economic factors in fishery models is a very important topic which is aimed to assesing fishery management and its economic consequences. The inclusion of economic factors in fichery models usually entails dealing with control theory (see the books by Cohen, 1987; Clark, 1990, and the works of Clarke and Munro, 1991, and Raïssi, 2001). Another approach considers as a new variable the price of the catch unit which depends on the actual amount of fish available on the market and on the costs. The article by Allen and Mc Glade (1986) shows a nice model describing the dynamics of a fish population with three age classes exploited by a fleet whose number of vessels can vary as a result of the variation of prices, the expected revenue of vessels and the costs of the haddock fishery. An explicit equation is incorporated in the model to describe the variation of the price of the catch unit. This model is able to predict cyclic variation of the fish population, price, number of vessels and catch. The extension to multispecies and multifleet spatial models is a very important goal of fishery models. However, such complete models incorporating many details are generally impossible to study analytically and only numerical simulations can be carried out.

This paper is situated in this general context and illustrates a fishery management model. We study fishing activity on two different spatial zones connected by migrations. In a previous work, Mchich et al. (2000) build a simple bioeconomical model which describes the stock-effort dynamic in a fishery with two adjacent zones, considering the 
migration rates of the fishing vessels to be constant. However, modern vessels can estimate the amount of fish present on a zone by various techniques (Gascuel et al., 1993), and, thus, when the catch is not large enough in a fishing area, they can rapidly leave this zone. Consequently, it is important to assume stock dependent vessels migration rates. The present model is aimed to study the effects of stock dependent decisions of vessels movements on the global dynamics of the fish stock and the fishing fleet size. We propose a model that takes into account spatial effects by distiguishing two fishing areas connected by fast stock dependent migrations of fishing vessels. Indeed, it is possible to think that very efficient fishing vessels, able to reach rapidly the most abundant areas, can lead to the extinction of the fish stock. The aim of this article is to study the effects of the efficiency of fishing vessels on the global dynamics of a fishery. Does it lead to extinction? Can the fishery be persistent? Does it induce large cyclic variations of the total fish stock and fishing vessels?

In Section 2 we present the model which consist in a system of four ordinary differential equations governing the two local fishing stocks and the number of vessels on each fishing area. The model includes two time scales, a fast one associated to quick movements between the fishing zones in comparison to a slow one corresponding to the growth of the fish population and the variation of the total number of vessels involved in the fishery. In Section 3 we proceed to analyse the model. Here we take advantage of the two time scales to reduce the dimension of the complete model by use of aggregation methods (see the review article on aggregation techniques by Auger and Bravo de la Parra, 2000). The aggregated model describes the dynamics of the total number of vessels and stocks and has the advantage that it is possible to perform its complete qualitative analysis. Section 4 develops a discussion of the results obtained in Section 3, and Section 5 introduces a control parameter in the model which enables the stabilization of the system avoiding the dangereous fluctuations that appear in some cases.

\section{Mathematical model}

We consider a model which describes the dynamics of two fish populations of densities $x_{1}$ and $x_{2}$ located at two different fishing zones, and exploited by two fishing fleets represented by their fishing efforts $E_{1}$ and $E_{2}$. To simplify, we may assume that all fishing vessels are identical and, consequently, the fishing efforts $E_{i}(t)$ on each area $i$ can be measured by the number of vessels present on this zone at time $t$.

We assume that population $x_{1}$, situated in the first zone, is harvested by fleet $E_{1}$, while population $x_{2}$, situated in the second zone, is harvested by fleet $E_{2}$.

We suppose that two processes occur at two different time scales. At the fast time scale, the total stock and the total number of vessels are constant. Thus, the fast part of the model only describes the displacement of fish and vessels between the two zones. The fact that migration is conservative yields that vessels and fish leaving a zone get into the other one.

At the slow time scale, the total fish stock and the number of vessels are not constant, the evolution of the stock and the effort in each of the two zones is represented by the stock-effort Schaefer model, also called Graham-Schaefer model (Schaefer, 1954). Regarding the fish stock, the slow part of the model describes, for each specific zone, the growth of the fish population according to the logistic model and its decrease due to the fishing effort. Concerning the effort, the number of vessels of the fishery increases or decreases if the revenue of the fishery is positive or negative, respectively. The revenue is the difference between the income and the cost. We assume constant prices for the caught and costs units on both fishing areas. This is a simplifying assumption as prices could depend on the abundance of fish available on the market at time $t$ (Allen and Mc Glade, 1986; Clark, 1990). We would like to investigate this process in a future contribution.

According to previous assumptions, the complete system reads as follows: 


$$
\left\{\begin{array}{l}
\dot{x}_{1}=R\left(k x_{2}-k^{\prime} x_{1}\right)+\left[r_{1} x_{1}\left(1-\frac{x_{1}}{K_{1}}\right)-q_{1} E_{1} x_{1}\right] \\
\dot{x}_{2}=R\left(k^{\prime} x_{1}-k x_{2}\right)+\left[r_{2} x_{2}\left(1-\frac{x_{2}}{K_{2}}\right)-q_{2} E_{2} x_{2}\right] \\
\dot{E}_{1}=R^{\prime}\left[m\left(x_{2}\right) E_{2}-m^{\prime}\left(x_{1}\right) E_{1}\right]+E_{1}\left(p_{1} q_{1} x_{1}-c_{1}\right) \\
\dot{E}_{2}=R^{\prime}\left[m^{\prime}\left(x_{1}\right) E_{1}-m\left(x_{2}\right) E_{2}\right]+E_{2}\left(p_{2} q_{2} x_{2}-c_{2}\right)
\end{array}\right.
$$

Parameters $r_{i}$ and $K_{i}(i=1,2)$ represent, respectively, the intrinsic growth rate and carrying capacity of the stock in zone $i$. The catchability coefficient of the fleet on zone $i(i=1,2)$ is $q_{i}$. The catchability coefficient is supposed to be constant and, for simplicity of calculations, we also assume $q_{i}=1, i=1,2$. Parameters $p_{i}$ and $c_{i}(i=1,2)$ are the price of the catch unit and the cost of the fishing effort unit, respectively, in zone $i$ and are assumed to be constant.

The constant coefficients $k$ and $k^{\prime}$ represent the fish per capita migration rates from zone 2 to zone 1 and from zone 1 to zone 2, respectively. The corresponding migration coefficients for the fishing vessels, $m^{\prime}\left(x_{1}\right)$ and $m\left(x_{2}\right)$, depend on the fish stock in the particular zone. We assume that:

$m^{\prime}\left(x_{1}\right)=\frac{1}{\alpha x_{1}+\alpha_{0}} \quad$ and $\quad m\left(x_{2}\right)=\frac{1}{\beta x_{2}+\beta_{0}}$

When $x_{i}$ increases, then $m\left(x_{i}\right)$ decreases. We can explain these rates of migration by the fact that the aim of the fleets owners is to increase their revenues. So, the fishing vessels try to operate in the most abundant zone. Consequently, the tendency of each fleet to leave a zone must increase when the stock decreases. We assume, without loss of generality and for simplicity of calculations, that $\alpha_{0}=\beta_{0}$ This can be justified by the fact that when the stock $\left(x_{1}\right.$ or $\left.x_{2}\right)$ is very small, then the rate of migration should be the same in both directions.

Since the fishing fleets are located in a border band of a small size the vessels of a fleet are assumed to move quickly between the two fishing zones and to operate on both of them in order to increase their revenue. Similarly, fish migrations between the two fishing zones are suppose to be fast. Thus, the motion of vessels and fish corresponds to a fast time scale, what is reflected in Eq.
(2.1) by parameters $R$ and $R^{\prime}$. The second term of each of the four equations of Eq. (2.1) is composed of two parts: the fast part which describes migrations and the slow part which stands for growth terms. This translates the fact that migrations are assumed to be fast in comparison with the growth processes. The fast parts include the dimensionless large $\left(R \gg 1\right.$ and $\left.R^{\prime} \gg 1\right)$ positive parameters $R$ and $R^{\prime}$ which are different but can be assumed to be equal by changing appropiately the migration parameters $k$ and $k^{\prime}$. So, henceforth we further assume that $R^{\prime}=R$.

In a previous work, Mchich et al. (2000), the authors studied the case of constant vessel migration rates, $m$ and $m^{\prime}$ being stock independent. Under some conditions, the qualitative behaviour of the model is summarized in the following two cases:

- The fish exploitation is not sustainable: the total fishing effort tends to zero and the fishing stock stabilizes at its carrying capacity.

- There is a durable fish exploitation at fixed globally stable fish stock and vessels number.

In this work, we investigate the effects of stock dependent vessel migration rates.

If we set $\varepsilon=\frac{1}{R}$, and $\tau=\frac{t}{\varepsilon}$, then Eq. (2.1) can be rewritten in the following form:

$$
\left\{\begin{array}{l}
\frac{\mathrm{d} x_{1}}{\mathrm{~d} \tau}=\left(k x_{2}-k^{\prime} x_{1}\right)+\varepsilon\left[r_{1} x_{1}\left(1-\frac{x_{1}}{K_{1}}\right)-E_{1} x_{1}\right] \\
\frac{\mathrm{d} x_{2}}{\mathrm{~d} \tau}=\left(k^{\prime} x_{1}-k x_{2}\right)+\varepsilon\left[r_{2} x_{2}\left(1-\frac{x_{2}}{K_{2}}\right)-E_{2} x_{2}\right] \\
\frac{\mathrm{d} E_{1}}{\mathrm{~d} \tau}=\left(m\left(x_{2}\right) E_{2}-m^{\prime}\left(x_{1}\right) E_{1}\right)+\varepsilon E_{1}\left(p_{1} x_{1}-c_{1}\right) \\
\frac{\mathrm{d} E_{2}}{\mathrm{~d} \tau}=\left(m^{\prime}\left(x_{1}\right) E_{1}-m\left(x_{2}\right) E_{2}\right)+\varepsilon E_{2}\left(p_{2} x_{2}-c_{2}\right)
\end{array}\right.
$$

where $\tau$ represents the fast time scale with respect to $t$. Eq. (2.3) is now in the form that allows its reduction by means of the so-called aggregation methods. 


\section{Results}

From the complete Eq. (2.3), we apply aggregation methods (Auger and Bravo de la Parra, 2000) to obtain a reduced system: a two dimensional system of ordinary differential equations governing the total fish stock $x(t)=x_{1}(t)+x_{2}(t)$ and the total fishing effort $E(t)=E_{1}(t)+E_{2}(t)$ at the slow time scale.

The sufficient conditions for a system to be perfectly as well as approximately aggregated have been investigated in the frame of general population models by Iwasa et al. (1987), Iwasa et al. (1989) and Levin and Pacala (1997). Some aggregation methods permit to reduce a system with a large number of variables involving different time scales into an aggregated system with a few global variables. The method is based on perturbation technics and on the application of an adequate version of the Center Manifold Theorem (Fenichel, 1971): see Poggiale (1994), Auger and Roussarie (1994) and Auger and Poggiale (1996), Michalski et al. (1997). The aggregation of the complete model consists in supposing that the fast dynamics has attained a stable equilibrium and in substituting this fast equilibrium into the equations of the complete model. The first step to achieve aggregation is to neglect the small terms of the order of $\varepsilon$ in Eq. (2.3) and to look for the existence of stable equilibria for its fast part.

\subsection{Fast equilibria}

We notice that $x(t)$ and $E(t)$, the global variables, are constants of motion of the fast process: migration. Fast equilibria are the solutions of the following system:

$$
\left\{\begin{array}{l}
0=k x_{2}-k^{\prime} x_{1} \\
0=k^{\prime} x_{1}-k x_{2} \\
0=m\left(x_{2}\right) E_{2}-m^{\prime}\left(x_{1}\right) E_{1} \\
0=m^{\prime}\left(x_{1}\right) E_{1}-m\left(x_{2}\right) E_{2}
\end{array}\right.
$$

and a simple calculation leads to the following result:

$$
\left(x_{1}^{*}, x_{2}^{*}, E_{1}^{*}, E_{2}^{*}\right)=\left(v_{1}^{*} x, v_{2}^{*} x, \eta_{1}^{*}(x) E, \eta_{2}^{*}(x) E\right)
$$

where:

$$
\left\{\begin{array}{l}
v_{1}^{*}=\frac{k}{k+k^{\prime}} \\
v_{2}^{*}=\frac{k^{\prime}}{k+k^{\prime}} \\
\eta_{1}^{*}(x)=\frac{\alpha v_{1}^{*} x+\alpha_{0}}{\left(\alpha v_{1}^{*}+\beta v_{2}^{*}\right) x+2 \alpha_{0}} \\
\eta_{2}^{*}(x)=\frac{\beta v_{2}^{*} x+\alpha_{0}}{\left(\alpha v_{1}^{*}+\beta v_{2}^{*}\right) x+2 \alpha_{0}}
\end{array}\right.
$$

The constants $v_{1}^{*}$ and $v_{2}^{*}$ represent the fast equilibrium proportions of stock on each patch, whereas $\eta_{1}^{*}(x)$ and $\eta_{2}^{*}(x)$ admit the same interpretation for the fishing effort. As we see there is a fast equilibrium for each pair of values of the global variables $x$ and $E$. These equilibria are easily proved to be stable for the fast dynamics.

\subsection{The aggregated system}

Coming back to the complete initial Eq. (2.3), we substitute the fast equilibria (Eq. (3.2)) and add the two fish stock and the two fishing effort equations. The state variables are replaced in terms of the fast equilibria as follows:

$x_{1}=v_{1}^{*} x, \quad x_{2}=v_{2}^{*} x, \quad E_{1}=\eta_{1}^{*}(x) E$,

$E_{2}=\eta_{2}^{*}(x) E$.

After some algebra, one obtains the following system of two equations governing the total fish stock and fishing effort variables at the slow time scale, that we call the aggregated model:

$\left\{\begin{array}{l}\dot{x}(t)=r x\left(1-\frac{x}{K}\right)-q(x) E x \\ \dot{E}(t)=E(p(x) x-c(x))\end{array}\right.$

where

$$
\left\{\begin{array}{l}
r=r_{1} v_{1}^{*}+r_{2} v_{2}^{*} \\
K=\frac{r}{\frac{r_{1}}{K_{1}}\left(v_{1}^{*}\right)^{2}+\frac{r_{2}}{K_{2}}\left(v_{2}^{*}\right)^{2}} \\
q(x)=v_{1}^{*} \eta_{1}^{*}(x)+v_{2}^{*} \eta_{2}^{*}(x) \\
p(x)=p_{1} v_{1}^{*} \eta_{1}^{*}(x)+p_{2} v_{2}^{*} \eta_{2}^{*}(x) \\
c(x)=c_{1} \eta_{1}^{*}(x)+c_{2} \eta_{2}^{*}(x)
\end{array}\right.
$$

The dynamics of Eq. (3.4) is a good approximation of the real dynamics of the global variables in 
the complete Eq. (3.2) if Eq. (3.4) is structurally stable, which is the case, and $\varepsilon$ is small enough, which is assumed.

Eq. (3.4) has nearly the same form as the slow parts of the complete system, but with parameters depending on global stock $x$ and the appearance of a term of catchability $q(x)$ in its first equation.

\subsection{Asymptotic behavior}

The $\dot{E}=0$ nullclines are two straight lines: $E=0$ and $x=x *$ The constant $x *$ is the unique positive solution of the equation $p(x) x-c(x)=0$ :

$$
\begin{aligned}
x^{*} & =\frac{\left(-\alpha_{0}\left(p_{1} v_{1}^{*}+p_{2} v_{2}^{*}\right)+c_{1} \alpha v_{1}^{*}+c_{2} \beta v_{2}^{*}\right)+\sqrt{\Delta_{*}}}{2\left(\alpha p_{1} v_{1}^{*^{2}}+\beta p_{2} v_{2}^{*^{2}}\right)} \\
& >0
\end{aligned}
$$

where:

$$
\begin{aligned}
\Delta_{*}= & \left(\alpha_{0}\left(p_{1} v_{1}^{*}+p_{2} v_{2}^{*}\right)-\alpha c_{1} v_{1}^{*}-\beta c_{2} v_{2}^{*}\right)^{2} \\
& +4 \alpha_{0}\left(c_{1}+c_{2}\right)\left(\alpha p_{1} v_{1}^{*^{2}}+\beta p_{2} v_{2}^{*^{2}}\right) \\
> & 0
\end{aligned}
$$

The $\dot{x}=0$ nullclines are $x=0$ and $r(1-x / K)-$ $q(x) E=0$. The latter could be explicitly expressed as:

$E=\frac{r\left(1-\frac{x}{K}\right)\left(\tau_{1} x+2 \alpha_{0}\right)}{\left(\tau_{2} x+\alpha_{0}\right)}$

where $\tau_{1}=\alpha v_{1}^{*}+\beta v_{2}^{*}$ and $\tau_{2}=\alpha v_{1}^{*^{2}}+\beta v_{2}^{*^{2}}$.

Eq. (3.4) has three equilibrium points: $(0,0),(K$, $0)$ and $\left(x^{*}, \frac{r}{q\left(x^{*}\right)}\left(1-x^{*} / K\right)\right)$, where $x^{*}$ is given by

Eq. (3.6). This last equilibrium belongs to the positive quadrant provided that $x^{*}<K$.

When $2 \tau_{2}<\tau_{1}$, the nontrivial $\dot{x}=0$ nullcline Eq. (3.8) has a maximum value in the positive quadrant, $\hat{E}$, and we denote $\hat{x}$ the corresponding fish stock value.

The Jacobian matrix $J(x, E)$ reads:

$$
J(x, E)=\left[\begin{array}{ll}
r-\frac{2 r x}{K}-q(x) E-q^{\prime}(x) x E & -q(x) x \\
E\left(p^{\prime}(x) x+p(x)-c^{\prime}(x)\right) & p(x) x-c(x)
\end{array}\right]
$$

In the Appendix $\mathrm{A}$, the stability properties of these equilibrium points are shown. The origin $(0$, 0 ) is always a saddle point. According to parameters values, we obtain the next five cases:

- If $2 \tau_{2}>\tau_{1}$ and $x^{*}>K$ then $\left(x^{*}, E^{*}\right)$ does not belong to the positive quadrant and $(K, 0)$ is a stable node, see Fig. 1.

- If $2 \tau_{2}>\tau_{1}$ and $x^{*}<K$ then $\left(x^{*}, E^{*}\right)$ belongs to the positive quadrant and is globally asymptotically stable while $(K, 0)$ is a saddle, see Fig. 2.

- If $2 \tau_{2}<\tau_{1}$ and $x^{*}>K$ then $\left(x^{*}, E^{*}\right)$ does not belong to the positive quadrant and $(K, 0)$ is a stable node, see Fig. 3 .

- If $2 \tau_{2}<\tau_{1}$ and $\hat{x}<x^{*}<K$ then $\left(x^{*}, E^{*}\right)$ belongs to the positive quadrant and is globally asymptotically stable while $(K, 0)$ is a saddle, see Fig. 4.

- If $2 \tau_{2}<\tau_{1}$ and $x^{*}<\hat{x}<K$ then $\left(x^{*}, E^{*}\right)$ belongs to the positive quadrant and is unstable. $(K, 0)$ is a stable node, see Fig. 5 . In this case, there exists a limit cycle.

Fig. 6 shows a suitable Poincaré-Bendixson box, see Arrowsmith and Place (1992). A trajectory starting from an initial point A (which should be chosen with $x>K$ and $E>\hat{E}$ ) is turning around the unstable positive nontrivial equilibrium $\left(x^{*}, E^{*}\right)$ and enters into the box at a point $B$. Any trajectory reaching the segment $A B$ at a point $M$ is entering into the box because the two

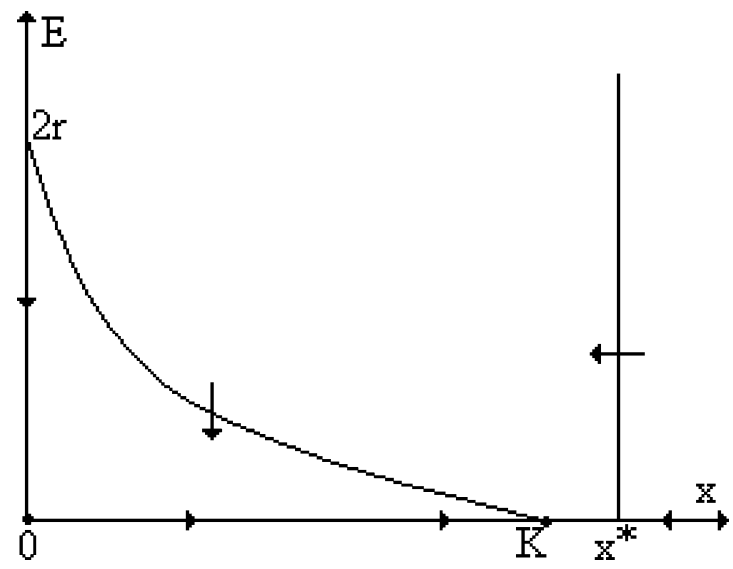

Fig. 1. Nullclines in the case $2 \tau_{2}>\tau_{1}$ and $x^{*}>K$. $\left(x^{*}, E^{*}\right)$ does not belong to the positive quadrant and $(K, 0)$ is a stable node. 


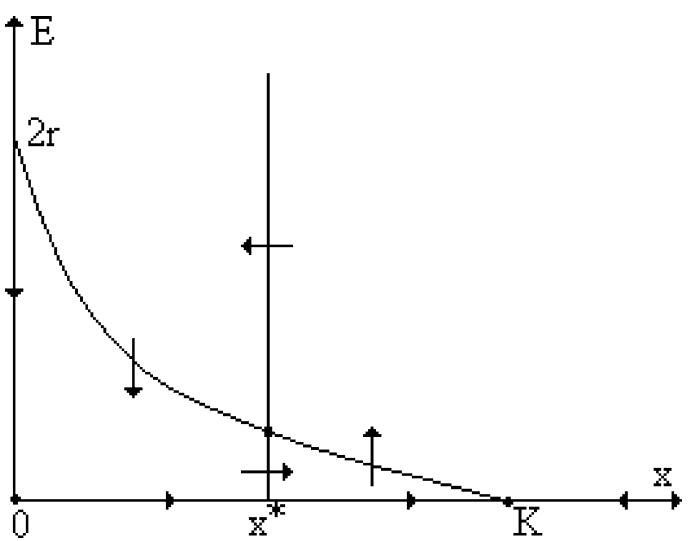

Fig. 2. Nullclines in the case $2 \tau_{2}>\tau_{1}$ and $x^{*}<K$. $\left(x^{*}, E^{*}\right)$ belongs to the positive quadrant and is globally asymptotically stable while $(K, 0)$ is a saddle.

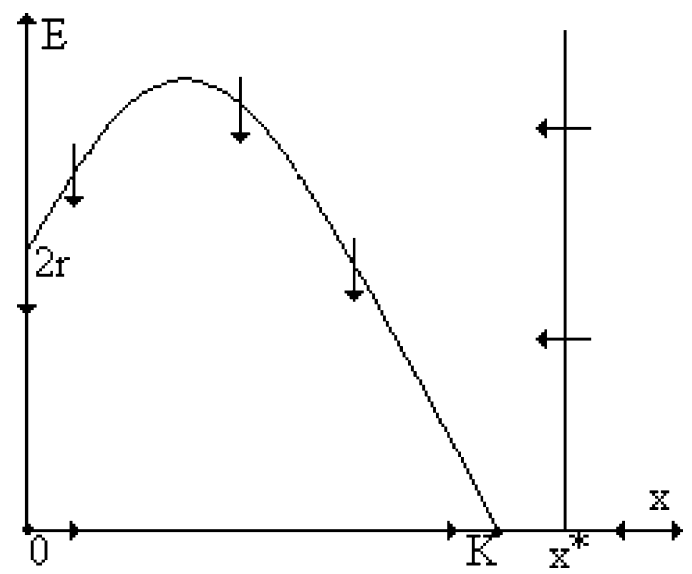

Fig. 3. Nullclines in the case $2 \tau_{2}<\tau_{1}$ and $x^{*}>K$. $\left(x^{*}, E^{*}\right)$ does not belong to the positive quadrant and $(K, 0)$ is a stable node.

components of its velocity are oriented towards the interior of the box. Any trajectory that enters the box cannot tend to the unique interior equilibrium $\left(x^{*}, E^{*}\right)$ which is unstable. Consequently, by use of the Poincaré-Bendixson theorem, this proves the existence of a limit cycle within the domain delimited by this box.

\section{Discussion of results}

Three cases will be discussed:

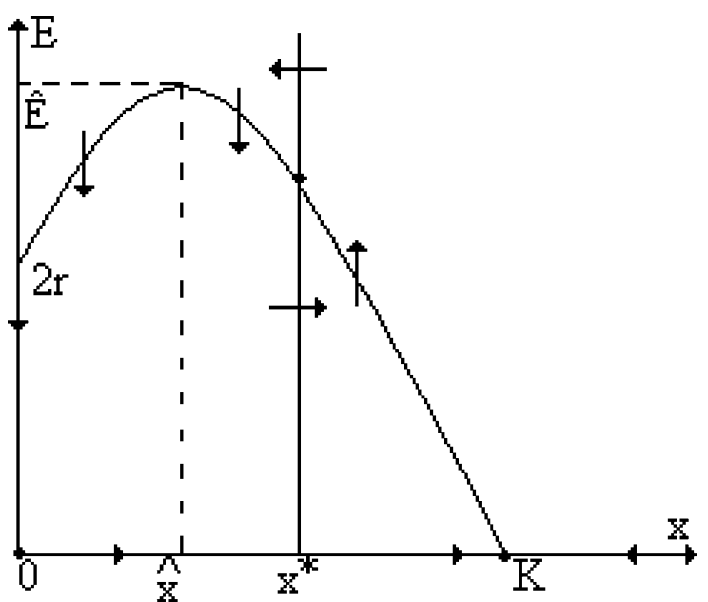

Fig. 4. Nullclines in ths case $2 \tau_{2}<\tau_{1}$ and $\hat{x}<x^{*}<K$. ( $\left.x^{*}, E^{*}\right)$ belongs to the positive quadrant and is globally asymptotically stable while $(K, 0)$ is a stable node.

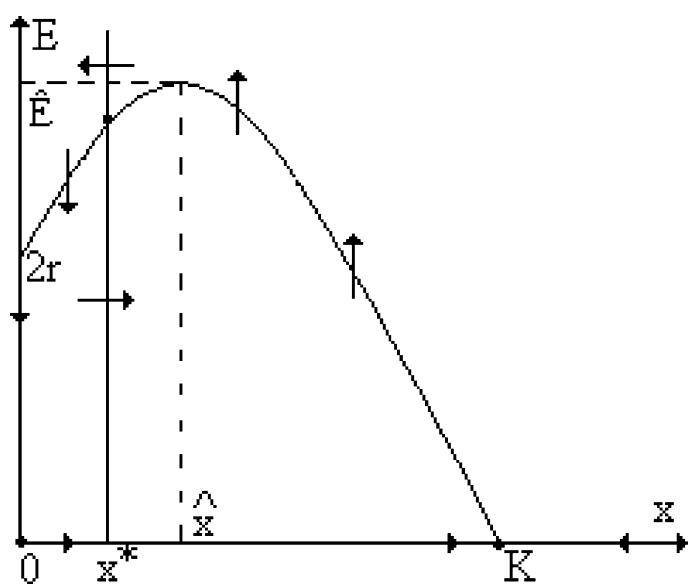

Fig. 5. Nullclines in the case $2 \tau_{2}<\tau_{1}$ and $x^{*}<\hat{x}<K$. $\left(x^{*}, E^{*}\right)$ belongs to the positive quadrant and is unstable. $(K, 0)$ is a stable node.

(1) Fig. 7 illustrates the case of fleets disappearence what entails the stabilization of the fish stock in its carrying capacity. From the point of view of the fishery, this case is not interesting because it does not allow a sustainable fishery exploitation.

(2) Fig. 8 presents the case where there is a positive equilibrium which is stable. In this case, in the long term, the total fishing effort approaches a constant equilibrium value $E^{*}$ as well as the biomass of the fish stock tends to a constant size 


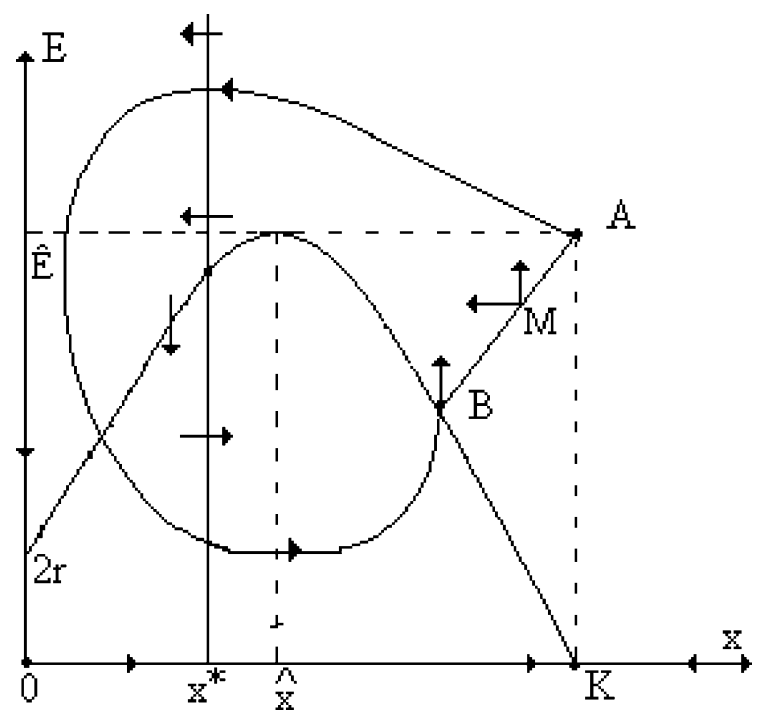

Fig. 6. Poincaré-Bendixson box. Any trajectory entering the box is trapped in this box, in which the unique equilibrium is unstable.

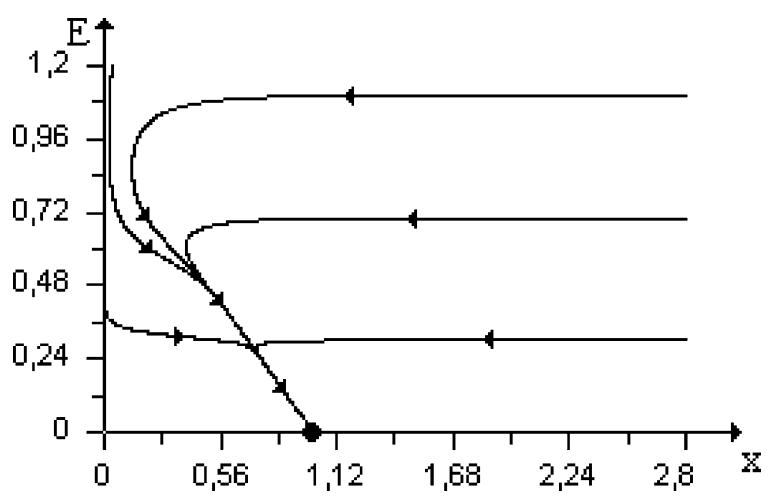

Fig. 7. Phase portrait in the case when the fishery is not sustainable. Parameters have been chosen as $\alpha=0.2 ; \beta=0.1$; $\alpha_{0}=1 ; p_{1}=0.025 ; p_{2}=0.02 ; c_{1}=0.02 ; c_{2}=0.015 ; K=1 ; r=$ $0.5 ; v_{1}=0.416667$ and $v_{2}=0.583333$.

$x^{*}<K$. This situation enables a permanent and durable fishery activity.

(3) An example of the case where the dynamics lead to stable limit cycle is shown in Fig. 9. In this case, the total fishing effort as well as the total fish stock are, in the long term, varying periodically. Fig. 10 shows the time variations of the total fish stock and of the total fishing effort with respect to time. These cyclical variations correspond to a

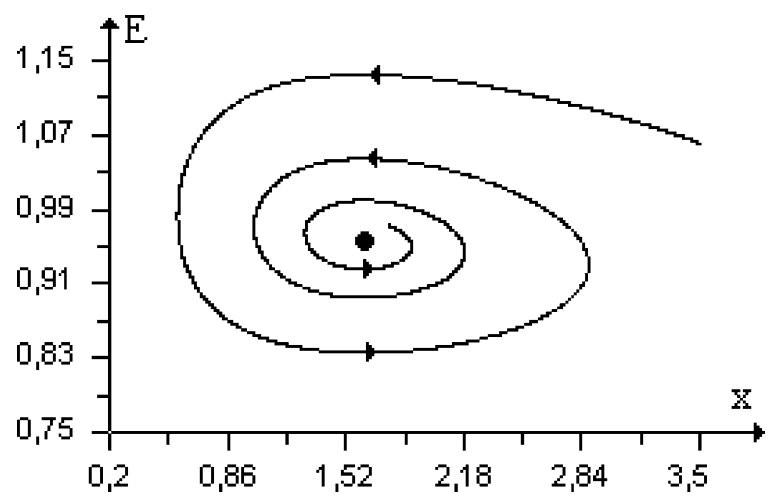

Fig. 8. Phase portrait in the case of a sustainable fishery. Parameters have been chosen as $\alpha=0.2 ; \beta=0.1 ; \alpha_{0}=1 ; p_{1}=$ $0.015 ; p_{2}=0.025 ; c_{1}=0.01 ; c_{2}=0.02 ; K=1000 ; r=0.5 ; v_{1}=$ 0.75 and $v_{2}=0.25$.

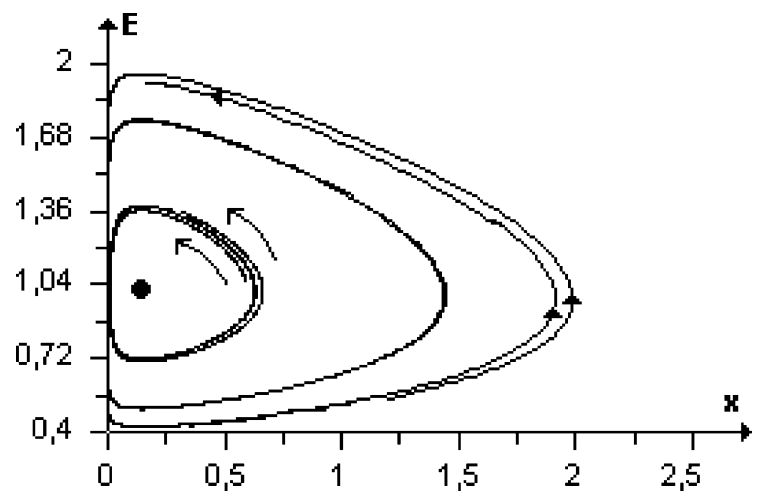

Fig. 9. Phase portrait in the case of stable limit cycle. Parameters have been chosen as $\alpha=0.4 ; \beta=0.2 ; \alpha_{0}=0.01$; $p_{1}=0.15 ; p_{2}=0.25 ; c_{1}=0.01 ; c_{2}=0.02 ; K=40 ; r=0.5 ; v_{1}=$ 0.416667 and $v_{2}=0.583333$.

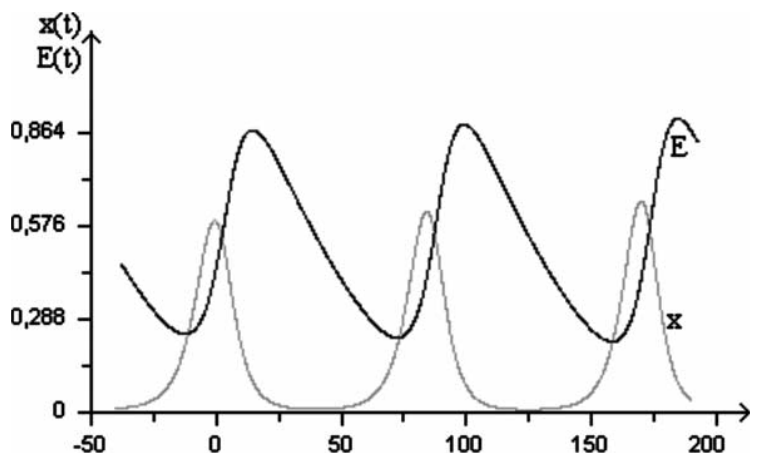

Fig. 10. Time variations of the total fish stock and fishing fleet in the case of the stable limit cycle (same parameters as in Fig. 9). 
long time period because they correspond to the dynamics of the aggregated model which approximately describes the dynamics of the system at the slow time scale.

The third case is interesting and new with respect to our previous study, (Mchich et al., 2000) in which no limit cycle occurred. In the present model, the limit cycle is the result of the stock dependence of the vessels migration rates. It is assumed that vessels leave rapidly the fishing zone with decreasing fish stock density because the catch income becomes too low. As a consequence, although the fish stock decreases a lot, it cannot go to extinction because, since the fishing fleets also decrease drastically, the fish stock can recover. Then, the fishery revenue increases and new vessels participate to the fishery, so starting a new cycle.

Belvéze (1984) has reported that the total sardine catch in the southern Moroccan sea has shown important fluctuations during the period of 1940-1983. The present study suggests that these fluctuations need not necessarily lead to extinction but could stabilize in a periodic variation merging periods of low and high fishery activity. This cyclic process of overexploiting periods followed by periods of recovering of the fishery activity should be a direct consequence of the efficiency of the fishery vessels. The model also suggests that the total process would be a long term process. The reason is that two types of processes are involved in the dynamics, fast ones associated with decisions of vessels to rapidly increase their captures and slow ones related to the demography of the fish population and the variations of the investment in the fleets.

\section{Introduction of a control variable}

As shown in the previous section, according to parameters values, the dynamics can lead to a stable equilibrium or to a stable limit cycle. From the point of view of a sustainable fishery, it is better to avoid important variations of the total fish stock and fishing effort as shown in Fig. 8. If the total stock density becomes too small for some period of stable cycle, environmental fluctuations could lead to the extinction of the stock. Also, if the number of fishing vessels decreases to a very small number that could practically represent the stop of the fishing activity. Thus, the case of a stable equilibrium is saver because it would maintain the stock and fishing effort close to a constant good level.

It appears therefore useful to introduce a control parameter in the model. This control parameter, which we call $u$, is a real constant, such that: $0<u<1$. A simple way for a coastal state to control its fishery is to limit the technical capacities of fishing vessels, i.e. by restricting vessels to use certain fishing techniques and, consequently to limit the catch of fleets. Such a decrease of vessels technical capacities could correspond to a decrease of catchability. At first, in our model, the catchabilities were set equal to 1 . Now, we add $u$ as a catchability term which is the same for the both fishing fleets. This can be done by multiplying the catch terms $E_{\mathrm{i}} x_{\mathrm{i}}$ by the parameter $u$ in all equations of the Eq. (2.3), what yields the following system:

$$
\left\{\begin{array}{l}
\frac{\mathrm{d} x_{1}}{\mathrm{~d} \tau}=\left(k x_{2}-k^{\prime} x_{1}\right)+\varepsilon\left[r_{1} x_{1}\left(1-\frac{x_{1}}{K_{1}}\right)-u E_{1} x_{1}\right] \\
\frac{\mathrm{d} x_{2}}{\mathrm{~d} \tau}=\left(k^{\prime} x_{1}-k x_{2}\right)+\varepsilon\left[r_{2} x_{2}\left(1-\frac{x_{2}}{K_{2}}\right)-u E_{2} x_{2}\right] \\
\frac{\mathrm{d}\left(E_{1}\right)}{\mathrm{d} \tau}=\left(m\left(x_{2}\right) E_{2}-m^{\prime}\left(x_{1}\right) E_{1}\right)+\varepsilon E_{1}\left(p_{1} u x_{1}-c_{1}\right) \\
\frac{\mathrm{d}\left(E_{2}\right)}{\mathrm{d} \tau}=\left(m^{\prime}\left(x_{1}\right) E_{1}-m\left(x_{2}\right) E_{2}\right)+\varepsilon E_{2}\left(p_{2} u x_{2}-c_{2}\right)
\end{array}\right.
$$

In that case, the aggregated system is:

$$
\left\{\begin{array}{l}
\dot{x}(t)=r x\left(1-\frac{x}{K}\right)-q(x) u E x \\
\dot{E}(t)=E(p(x) u x-c(x))
\end{array}\right.
$$

The study of this system is straightforward.

The Jacobian matrix $J\left(x^{*}, E^{*}\right)$ becomes:

$$
\begin{aligned}
& J\left(x^{*}, E^{*}\right)= \\
& {\left[\begin{array}{ll}
-\frac{r x^{*}}{K}-u q^{\prime}\left(x^{*}\right) x^{*} E^{*} & -u q\left(x^{*}\right) x^{*} \\
E^{*}\left(u p^{\prime}\left(x^{*}\right) x^{*}+u p\left(x^{*}\right)-c^{\prime}\left(x^{*}\right)\right) & 0
\end{array}\right]}
\end{aligned}
$$


with the trace:

$\operatorname{tr} J\left(x^{*}, E^{*}\right)=-\frac{r x^{*}}{K}-u \frac{\alpha_{0}\left(2 \tau_{2}-\tau_{1}\right)}{\left(\tau_{1} x^{*}+2 \alpha_{0}\right)^{2}} x^{*} E^{*}$

In the case $2 \tau_{2}<\tau_{1}$, in order to have $\operatorname{tr} J\left(x^{*}\right.$, $\left.E^{*}\right)<0$, what assures the existence of a stable equilibrium $\left(x^{*}, E^{*}\right)$, it is necessary to maintain the control parameter under a certain threshold value:

$0<u<\frac{r\left(\tau_{1} x^{*}+2 \alpha_{0}\right)^{2}}{K E^{*} \alpha_{0}\left(\tau_{1}-2 \tau_{2}\right)}$

Thus, when $T=r\left(\tau_{1} x^{*}+2 \alpha_{0}\right)^{2} / K E^{*} \alpha_{0}\left(\tau_{1}-2 \tau_{2}\right)$ is larger than 1 , the equilibrium $\left(x^{*}, E^{*}\right)$ is always stable without control. On the other hand, when $T<1$ it is necessary to control the system by setting the parameter $u$ as in Eq. (5.5).

\section{Conclusion}

In this contribution, we generalized a previous work(Mchich et al., 2000), where the authors build a similar dynamical model but in the simplest case of stock independent vessels migration rates. Two different cases are distinguished: extinction of the fishery and coexistence of the fish stock and the fishing fleet at a constant stable equilibrium.

In the present work, we investigate the effects of stock dependent vessels migration rates. Apart from the two above mentioned cases, extinction and coexistence, it appears a new case with a stable limit cycle. This model shows that different strategies for fishing at a fast time scale can have important consequences on the global evolution of the fishery in the long term. However, this new situation can lead to risk of extinction and we show that it is possible to avoid this risk by controlling the fishing efforts. A future work would consist on using time dependent control variables, and also to use stock dependent prices and costs.

Moroccan coast is $3500 \mathrm{~km}$ long with several important fishing zones. Fishing vessels can move from north to south to exploit different fish species and also they can operate either on coastal or high sea fisheries. So, another situation worth to be considered is that of a spatial network of several zones connected by migrations. In the case of fast migrations, the aggregated model give us good qualitative informations about the dynamics of the total fish stock and fleet size in the long term.

\section{Acknowledgements}

This work has been partially supported by Proyecto de Investigaciûn PB98-0702 (Spanish M.E.C.). We thank the anonymous referee for the valuable comments that allowed us to improve the manuscript. We thank Olivier Maury for finding some references that are related to this study. We also thank Jean-Christophe Poggiale for his comments on the mathematical part of the article.

\section{Appendix A}

The Jacobian matrix $J(x, E)$ reads:

$J(x, E)=\left[\begin{array}{ll}r-\frac{2 r x}{K}-q(x) E-q^{\prime}(x) x E & -q(x) x \\ E\left(p^{\prime}(x) x+p(x)-c^{\prime}(x)\right) & p(x) x-c(x)\end{array}\right]$

(a) At the point $(0,0)$, the Jacobian matrix:

$J(0,0)=\left[\begin{array}{ll}r & 0 \\ 0 & -\frac{c_{1}+c_{2}}{2}\end{array}\right]$

has two real eigenvalues with opposite signs and thus $(0,0)$ is a saddle point.

(b) At the point $(K, 0)$, the Jacobian matrix:

$J(K, 0)=\left[\begin{array}{ll}-r & -q(K) K \\ 0 & p(K) K-c(K)\end{array}\right]$

has two real eigenvalues, one is negative $\lambda_{1}=-r$ and $\lambda_{2}=p(K) K-c(K)$. Two cases appear:

- if $x^{*}<K$, then $\lambda_{2}>0$ and $(K, 0)$ is a saddle point.

- if $x^{*}>K$, then $\lambda_{2}<0$ and $(K, 0)$ is a stable node. 
(c) At the point $\left(x^{*}, \frac{r}{q\left(x^{*}\right)}\left(1-\frac{x^{*}}{K}\right)\right)$, the Jacobian matrix $J\left(x^{*}, E^{*}\right)$ becomes:

$$
\begin{aligned}
& J\left(x^{*}, E^{*}\right) \\
& =\left[\begin{array}{ll}
-\frac{r x^{*}}{K}-q^{\prime}\left(x^{*}\right) x^{*} E^{*} & -q\left(x^{*}\right) x^{*} \\
\left(E^{*}\left(p^{\prime}\left(x^{*}\right) x^{*}+p\left(x^{*}\right)-c^{\prime}\left(x^{*}\right)\right)\right. & 0
\end{array}\right]
\end{aligned}
$$

where

$$
\begin{aligned}
\operatorname{det} & J\left(x^{*}, E^{*}\right) \\
= & \frac{q\left(x^{*}\right) x^{*} E^{*}}{\left(\tau_{1} x^{*}+2 \alpha_{0}\right)^{2}} \\
& \times\left(\tau_{1}\left(\alpha p_{1} v_{1}^{*^{2}}+\beta p_{2} v_{2}^{*^{2}}\right) x^{* 2}+2 \alpha_{0} \sqrt{\Delta_{*}}\right. \\
& \left.\quad+\alpha_{0} \tau_{1}\left(c_{1}+c_{2}\right)\right) \\
\succ & 0
\end{aligned}
$$

and $\Delta_{*}$ is given by Eq. (3.7).

On the other hand, we have:

$\operatorname{tr} J\left(x^{*}, E^{*}\right)=-\frac{r x^{*}}{K}-\frac{\alpha_{0}\left(2 \tau_{2}-\tau_{1}\right)}{\left(\tau_{1} x^{*}+2 \alpha_{0}\right)^{2}} x^{*} E^{*}$

It is straightforward to see that $\operatorname{tr} J\left(x^{*}, E^{*}\right)<0$ whenever $2 \tau_{2}>\tau_{1}$, what yields the asymptotic stability of $\left(x^{*}, E^{*}\right)$.

When $2 \tau_{2}<\tau_{1}$, two different cases happen:

$-x^{*}>\hat{x}$, then $\operatorname{tr} J\left(x^{*}, E^{*}\right)<0$ and thus $\left(x^{*}, E^{*}\right)$ is asymptotically stable.

$-x^{*}<\hat{x}$, then $\operatorname{tr} J\left(x^{*}, E^{*}\right)>0$ and thus $\left(x^{*}, E^{*}\right)$ is unstable.

This can be proved by showing that the trace can be rewritten as:

$$
\begin{aligned}
& \operatorname{tr} J\left(x^{*}, E^{*}\right)=r x^{*} \\
& \times \frac{\left(\tau_{1} \tau_{2}\left(x^{*}\right)^{2}+2 \alpha_{0} \tau_{1} x^{*}+\alpha_{0}\left(2 \alpha_{0}+2 K \tau_{2}-K \tau_{1}\right)\right)}{K\left(\tau_{1} x^{*}+2 \alpha_{0}\right)\left(\tau_{2} x^{*}+\alpha_{0}\right)}
\end{aligned}
$$

and then that its sign changes when $x^{*}$ is smaller or larger than $\hat{x}$.

\section{References}

Allen, P.M., Mc Glade, J.M., 1986. Dynamics of discovery and exploitation: the case of the scotian shelf groundfish fisheries. Canadian Journal of Fish Aquatic Science 43, 11871200.

Arrowsmith, D.K., Place, C.M., 1992. Dynamical Systems. Differential Equations, Maps and Chaotic Behaviour. Chapman and Hall, London.

Auger, P.M., Bravo de la Parra, R., 2000. Methods of aggregation of variables in population dynamics. Comptes Rendus de l'Académie des Sciences, série III Paris, 323, 665-674.

Auger, P.M., Poggiale, J.C., 1996. Emergence of population growth models: fast migration and slow growth. Journal of Theoretical Biology 182, 99-108.

Auger, P.M., Roussarie, R., 1994. Complex ecological models with simple dynamics: from individuals to population. Acta Biotheoretica 42, 111-136.

Beddington, J.R., May, R.M., 1977. Harvesting natural populations in a randomly fluctuating environment. Science 197, $463-465$.

Belvéze, H., 1984. Biologie et Dynamique des Populations de Sardine (Sardina Pilchardus Walbaum) Peuplant les CÙtes Atlantiques Marocaines et Propositions pour un Aménagement des PÍcheries. PhD thesis at the Bretagne Occidentale University.

Clark, C.W., 1990. Mathematical Bioeconomics: The Optimal Management of Renewable Resources, Second ed.. A Wiley-Interscience.

Clarke, F.H., Munro, G.R., 1991. Coastal states, distant water fishing nations and extended jurisdiction: conflicting views of the future. Natural Resource Modelling 5, 345-369.

Cohen, Y. (Ed.), Applications of Control Theory in Ecology. Lecture Notes in Biomathematics, vol. 73. Springer, Berlin, Heidelberg, New York, Tokyo 1987.

Fenichel, N., 1971. Persistence and smoothness of invariant manifolds for flows. Indiana University Mathematical Journal 21, 193-226.

Gascuel, D., Fonteneau, A., Foucher, E., 1993. Analyse de l'Evolution des Puissances de PÍche par l'Analyse des Cohortes: Application aux Senneurs Exploitant l'Albacore (Thunnus Albacares) dans l'Atlantique Est. Aquatic Living Resources 6, 15-30.

Haddon, M., 2001. Modelling and Qualitative Methods in Fisheries. CRC Press, New York.

Iwasa, Y., Andreasen, V., Levin, S.A., 1987. Aggregation in model ecosystems I. Perfect aggregation. Ecological Modelling 37, 287-302.

Iwasa, Y., Levin, S.A., Andreasen, V., 1989. Aggregation in model ecosystems II. Approximate aggregation. IMA Journal of Mathematics Applied in Medicine and Biology 6, 1-23.

Levin, S., Pacala, S., 1997. Theories of simplification and scaling of spatially distributed processes. In: Tilman, D., Kareiva, P. (Eds.), Spatial Ecology: The Role of Space in 
Population Dynamics and Interspecific Interactions. Princeton University Press, Princeton, pp. 204-232.

Mackinson, S., 2000. An adaptative fuzzy expert system for predicting structure, dynamics and distribution of herring shoals. Ecological Modelling 126, 155-178.

Mchich, R., Auger, P.M., Raïssi, N., 2000. The dynamics of a fish stock exploited between two fishing zones. Acta Biotheoretica 48 (3/4), 207-218.

Michalski, J., Poggiale, J.C., Arditi, R., Auger, P.M., 1997. Macroscopic dynamic effects of migrations in Patchy Predator-Prey systems. Journal of Theoretical Biology 185, 459-474.

Millischer, L., Gascuel, D., Biseau, A., 1999. Estimation of the overall fishing power: a study of the dynamics and fishing strategies of Brittany's industrial fleets. Aquatic Living Resources 12 (2), 89-103.

Poggiale, J.C., 1994. Applications des Variétés Invariantes à la Modélisation de l'Hétérogénéité en Dynamique des Populations. $\mathrm{PhD}$ thesis at Bourgogne University, Dijon.
Quinn, T.J., Deriso, R.B., 1999. Quantitative Fish Dynamics. Oxford University Press Inc, New York.

Raïssi, N., 2001. Features of bioeconomics models for the optimal management of a fishery exploited by two different fleets. Natural Resource Modeling 12 (2), 1-24.

Rotenberg, M., 1987. Effect of certain stochastic parameters on extinction and harvested populations. Journal of Theoretical Biology 124, 455-472.

Schaefer M.B., 1954. Some aspects of the dynamics of populations important to the management of the commercial marine fisheries. Bulletin of the Inter-American Tropical Tuna Commission 1, pp. 27-56

Vasconcellos, M., Mackinson, S., Sloman, K., Pauly, D., 1997. The stability of trophic mass-balance models of marine ecosystems: a comparative analysis. Ecological Modelling $100,125-134$. 